\title{
Histoire des sciences Frédéric Joliot-Curie et la première pile atomique française
}

Pierre Radvanyi (radva@ipno.in2p3.fr)

Institut de physique nucléaire, Université Paris-Sud, 91406 Orsay Cedex

Frédéric Joliot est décédé

il y a 50 ans. Avec Irène

Joliot-Curie, il avait découvert

en 1934 la radioactivité

artificielle. En 1939-1940,

aussitôt après la découverte

de la fission de l'uranium,

il étudie, avec Halban et

Kowarski, la possibilité puis

les conditions de réalisation

d'une réaction en chaîne.

Des brevets sont pris

et des contacts établis avec

l'industrie ; un commando

en Norvège permet d'obtenir

l'eau lourde. L'invasion

de la France interrompt

les efforts. Ils seront repris

après-guerre avec la création

du CEA et la mise en route

en 1948 de Z0É, première

pile atomique française.

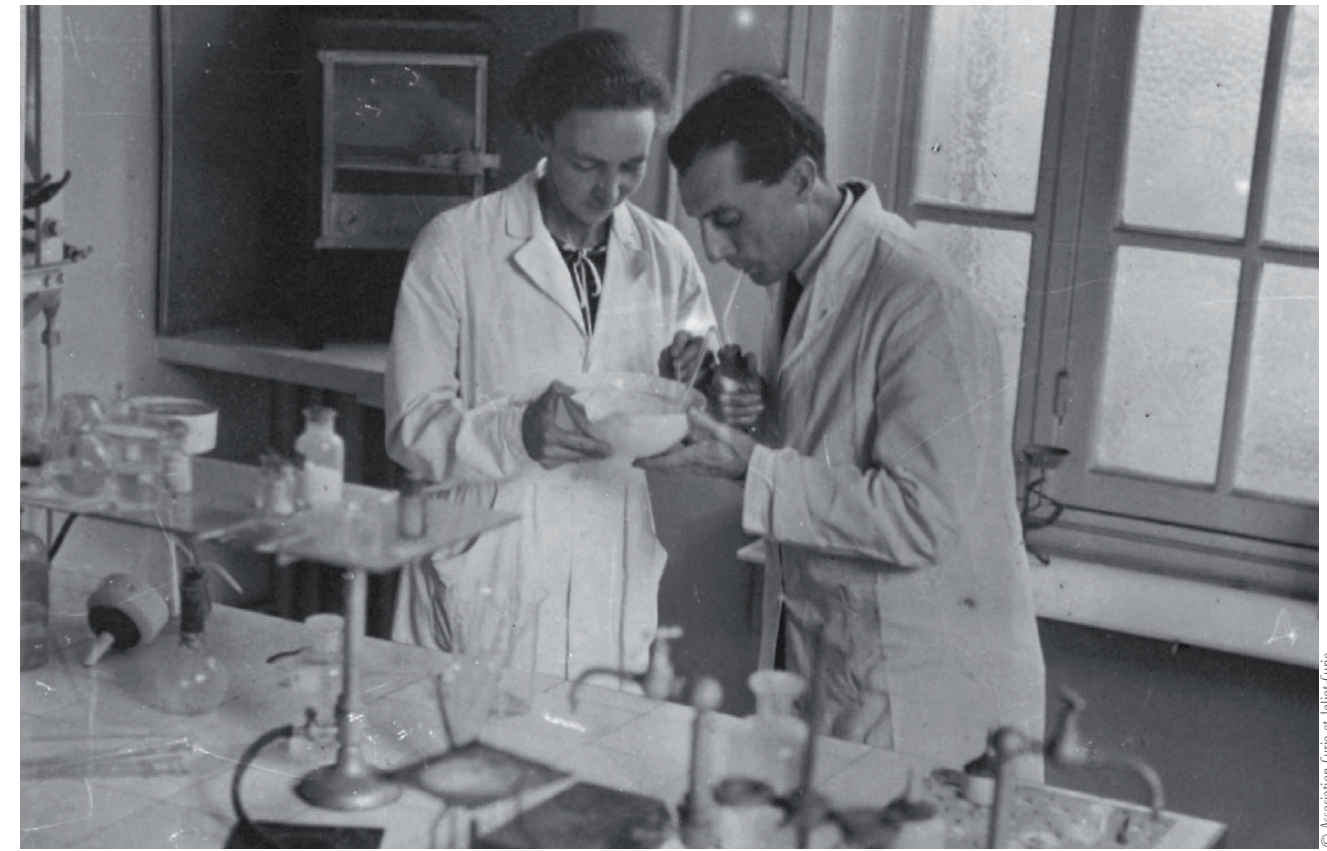

Irène et Frédéric Joliot-Curie en 1932, dans leur laboratoire de physique à I'Institut du Radium.

L'année 2008 marque le cinquantième anniversaire de la mort de Frédéric Joliot (19001958), ainsi que le soixantième anniversaire de la mise en route, sous sa direction, du premier réacteur nucléaire français, ZOÉ.

Frédéric Joliot est né à Paris le 19 mars 1900. Sorti major de l'École municipale de physique et chimie industrielles, il est présenté par le directeur des études, Paul Langevin, à Marie Curie, qui le recrute aussitôt comme préparateur particulier dans son laboratoire de l'Institut du Radium. Irène Curie, fille de Marie et de Pierre, est chargée de le piloter. Les deux jeunes gens se marieront en 1926 et entreprendront une série d'expériences en commun dans le domaine de la radioactivité.

\section{Découverte de la radioactivité artificielle}

En 1930, à Berlin, W. Bothe et H. Becker constatent que le bombardement du béryllium par des particules alpha produit un rayonnement très pénétrant non identifié. Frédéric et Irène réalisent de petites sources très intenses de polonium, émetteur alpha. Au début de 1932, ils observent que le rayonnement pénétrant est capable de projeter des protons de recul hors de feuilles de substances hydrogénées. Ce sera le point de départ de James Chadwick qui, un mois plus tard, à Cambridge, découvre que le rayonnement inconnu est constitué de "neutrons".

Poursuivant leurs expériences, Frédéric et Irène découvrent, en janvier 1934, la " radioactivité artificielle ", c'est-à-dire l'existence d'isotopes radioactifs d'éléments stables formés par des réactions nucléaires dans d'autres éléments (par exemple du phosphore radioactif dans l'aluminium). C'est une généralisation remarquable de la radioactivité naturelle avec de multiples applications, en particulier en biologie. Cette découverte vaudra aux deux jeunes chercheurs le prix Nobel de chimie de 1935. $\mathrm{Au}$ retour de Stockholm, lors d'un banquet en l'honneur des nouveaux lauréats, Paul Langevin propose de les appeler dorénavant les « Joliot-Curie».

Aussitôt, à Rome, Enrico Fermi et son équipe se mettent en devoir de produire des isotopes radioactifs de tous les éléments chimiques en utilisant des neutrons, capables de pénétrer dans les noyaux les plus lourds. Ils observent dans l'uranium irradié par des neutrons ce qu'ils pensent être des "transuraniens ", des noyaux plus lourds que l'uranium. Le flambeau est repris à Berlin par Otto Hahn, Lise Meitner et 
MINISTERE

DE L'WDUSTRIE ET DU COMMERCE BREVET D'INVENTION

SERVICE

Gr. 5. - C. 2 .

N" 976.541

1. PROPRIÉTÉ INDUSTRIELLE

Dispositif de production d'énergie.

CAISSE NATIONALE DE LA RECHERCHE SCIENTIFIQUE résidant

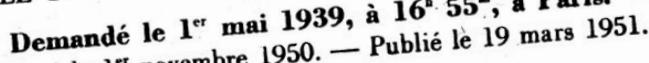

Délivré le $l^{\text {ex }}$ novembre 1950 journée en exécution de l'article $11, \oint 7$, (Brevet d'invention dont la délivrance a été ajournée en exécution de lo lo 7 avril 1902:)
de la loi du 5 juillet 1844 modifiée par la loi 7 a

Un sait que l'absorption d'un neutron par un Un au d uranum peut provoquer la rupture de ce

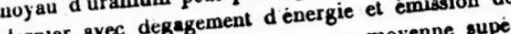
dernier avec degafente moyenne supenouveaux neutrons ea nombre ea me ainsi émis, un rieur a l'unite. Parmi les neutrons ains exrovoquer certain nombre peuvent a leur lous pouvelles rupcertur des noyaux d'uranium - de nouvelles pour- our des noptures de noyaux d'uranium pourtures, et les ruptures de noys suivant une progresront ainai aller en croissant suivant de quantité sion gémétrique, avec dénergic.

extrémement considérables denergic.

On sest rendu compte, conformément a la pré onte invention, que si l'on parvenait à provoquer sente invention, que sich d'une masse linitée d'uranium (ou de compose d'uranium ou d'un melan de nium (ount de l'uranium), on pourratrielles l'énercontenant de utiliser à des fins industrielles lichers gie ainai développée par les chaines de ruphares

succesaives.
Main on se beurte immédiatement à une diff.

successives.
Main on se heurte immédiatement à une diffiMais on se heurte immes chaînes pouvant re raminer d'une manière illimitée, la réaction peut denes les

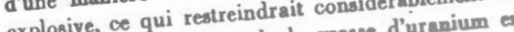
cxplosive, ce quilination de la masse duranio possibilités d'utalisation de la mable d'énergie indus. question

triell. On a donc cherché à maitriser le dégagemert d'énergie en l'empêchint bu lide ì cet effet

épartitions des constituants do dispositif, arrétant chaines: insi le développement des dans le dispositif un ou 2. Ensuite, d'introduire dans le corps simples ou plusieurs ćlérnents - a létat - susceptibles d'ab de combinaisons chimiques - suscertion d'autant orber les neutrons ralentis en proportion l'uranium plus forte par rapport à l'absorption

que la température est plus éleve. ou l'emploi conPar le premier de ces moyens chaines peuvent de jugué de ces deux mojens, les chargie suffisamment développer jusqu'à ce qu'une énergie sutomatiquo deve soit libérée, et être slors automatiqu b. ment interrompues ou limitces, evion. ment interromesplosif de la réaction. d'uranium développement explosil dérer de la masse d'uraniuna On arrive ainsi a le son utilisation induatrielle, considéréc, en vue de son utilisalion l'énergie qu'cl

et au fur et ì mesure des besoins, lentations. et a fareptible de fournir par transule partie des est susceptide da vitesse de tout ou parte manese Pour reduire la introduit au sein de la mase neutrons émis, on intent une répartition qui a'ent d'uranium - et suivant un - des éléments trè pas nécenaairement uniforme dentérium par exem pas no que l'hydrogene, le dentéléments légers

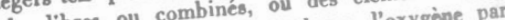
the que le glucinium, le carbone

le combines. Un avantege opieial des ralentisbeurs tres legers

Première page du brevet déposé le $1^{\text {er }}$ mai 1939.

Fritz Strassmann ; dans leurs publications, ces derniers indiquent alors avoir identifié plusieurs séries de transuraniens. Dans les premiers mois de 1938, à Paris, Irène Joliot-Curie et Paul Savitch ouvrent une nouvelle voie d'étude du problème.

\section{Fission, réaction en chaîne et contexte international}

En juillet 1938, à la suite de l'Anschluss, Lise Meitner, physicienne autrichienne d'origine juive, doit fuir l'Allemagne et se réfugier en Suède. Hahn et Strassmann reprennent leurs séparations chimiques pour examiner avec plus de rigueur encore si certains transuraniens ne seraient pas en fait des isotopes du radium. Peu avant Noël 1938, à leur grande surprise, ils découvrent que ces radioéléments ne sont pas des isotopes du radium, mais du baryum, élément beaucoup plus léger ; des noyaux d'uranium auraient donc "éclaté " sous l'action des neutrons. Leurs résultats seront publiés début janvier 1939. Alertée par Otto Hahn, Lise Meitner, avec son neveu Otto Frisch, donne une explication physique du phénomène, auquel ce dernier donnera le nom de "fission ".
Professeur au Collège de France, Joliot se saisit aussitôt du problème. Il se rend compte, comme plusieurs de ses collègues à travers le monde, que la fission doit libérer une énergie considérable. Cette constatation le conduit immédiatement à une expérience sur les noyaux de recul, apportant une preuve physique de la fission. Un cliché de chambre à brouillard lui apporte une confirmation supplémentaire. Il pense que la fission doit être accompagnée de l'émission de nouveaux neutrons, ce qui rendrait possible une réaction en chaine. Il forme alors une équipe avec Hans Halban et Lew Kowarski pour vérifier par l'expérience que de nouveaux neutrons sont effectivement émis, pour mesurer ensuite leur énergie et déterminer leur nombre. Les résultats obtenus sont très encourageants.

L'équipe du Collège de France est en compétition avec une équipe de l'université Columbia de New York, conduite par Fermi, à laquelle se joint Leo Szilard. L'équipe française est en général en avance d'une à trois semaines ; elle demande à Francis Perrin de se joindre à elle pour calculer les dimensions d'un dispositif producteur d'énergie. Les $1^{\mathrm{er}}, 2$ et 4 mai 1939, elle dépose trois demandes de brevets au nom de la Caisse nationale de la recherche scientifique (ancêtre du CNRS). Aussitôt après, F. Joliot se rend à Bruxelles pour proposer une collaboration industrielle à l'Union minière du Haut-Katanga. Un accord est signé et un projet de convention est préparé. L'Union minière met un total de 8 tonnes d'oxyde d'uranium à la disposition de l'équipe, qui poursuit ses travaux au laboratoire de synthèse atomique à Ivry. En quelques semaines, F. Joliot est passé de la recherche fondamentale à la recherche appliquée. Aux États-Unis, N. Bohr et J.A. Wheeler montrent que l'uranium 235 , isotope peu abondant de l'uranium, est responsable de la fission par neutrons lents.

La guerre éclate le $1^{\text {er }}$ septembre 1939. Les travaux se poursuivent, mais leurs résultats ne sont plus publiés ; un pli cacheté est déposé à l'Académie des sciences. Frédéric Joliot est reçu par Raoul Dautry, le nouveau ministre de l'Armement, et lui explique les objectifs des travaux en cours. Les neutrons lents sont plus efficaces pour provoquer des fissions ; l'équipe utilise un milieu hydrogéné (modérateur) pour ralentir les neutrons. Elle se rend compte cependant que, pour obtenir une réaction en chaîne divergente, modérateur et uranium doivent être séparés pour former un dispositif hétérogène. Les physiciens constatent que l'hydrogène ordinaire absorbe trop les neutrons et ils souhaitent alors utiliser du deutérium comme modérateur. Il leur faut donc obtenir de l'eau lourde qui n'est préparée à l'époque qu'à Rjukan en Norvège. S'appuyant sur un rapport de Joliot, Dautry décide d'envoyer en mission secrète en Norvège le lieutenant Jacques Allier, à la tête d'un commando, pour obtenir ce précieux produit. L'opération se déroule avec succès au début de mars 1940, permettant de ramener en France 167 litres d'eau lourde.

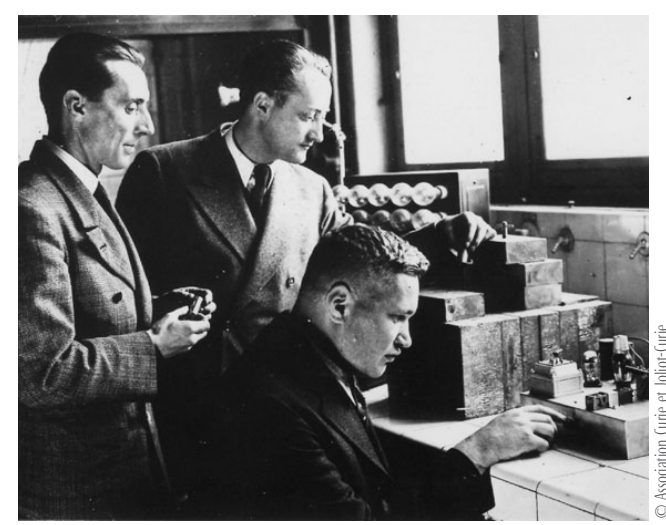

Joliot (à gauche), Halban (au centre) et Kowarski (à droite, ajustant un amplificateur d'impulsions) au laboratoire de chimie nucléaire du Collège de France. 
Deux nouveaux brevets sont pris les 30 avril et $1^{\text {er }}$ mai 1940 . Mais, peu après, commence l'invasion allemande. Halban, Kowarski, l'eau lourde et une grande partie des appareils sont repliés à Clermont-Ferrand, que F. Joliot rejoint juste avant l'entrée de la Wehrmacht à Paris. La situation est devenue dramatique. Les trois physiciens emmènent l'eau lourde à Bordeaux. À la demande de Joliot, Halban et Kowarski, munis d'un ordre de mission, embarquent le 17 juin pour l'Angleterre à bord du "Broompark", emportant le stock d'eau lourde et les documents correspondant aux derniers résultats obtenus. F. Joliot décide de rester en France. L'uranium, transporté au Maroc, est caché dans une galerie de mine désaffectée pendant toute la guerre. Parvenus à Londres, Halban et Kowarski rédigent le résumé et les conclusions des derniers travaux de l'équipe ; ils terminent par les phrases suivantes : "Deux voies sont préconisées pour la production d'énergie : la méthode des neutrons lents avec un petit enrichissement en uranium 235 ; ou l'espoir que la capture de neutrons par l'uranium 238 conduise en fin de compte à un nouveau noyau fissile ${ }^{(1)}$."

Frédéric Joliot, revenu à Paris, fait face. Il trouve son laboratoire du Collège occupé par les Allemands, mais placé sous l'autorité d'un physicien ami, Wolfgang Gentner, un " ancien » de l'Institut du Radium. Celui-ci le protégera efficacement, alors que Joliot s'engage activement dans la Résistance. Le physicien français entreprend des recherches en collaboration avec des biologistes sur l'utilisation des radioéléments en biologie.

(1) Ce sera en effet le plutonium 239, découvert à Berkeley en 1940/1941.

(2) L'espace ZOÉ du CEA/Fontenay-aux-Roses peut se visiter sur rendez-vous. Contact : 0146549600 ou 0146547080 .

\section{Après la Libération: le CEA et ZOÉ}

À la Libération, en août 1944, Joliot est nommé directeur général du CNRS. Il apprend peu à peu ce qui a été réalisé aux États-Unis pendant la guerre, et notamment la mise en route de la première pile atomique (réacteur nucléaire) à Chicago, sous la direction de Fermi, en décembre 1942. Joliot a deux entretiens avec le général de Gaulle, président du gouvernement provisoire, en novembre 1944 et, en compagnie de Pierre Auger, en mai 1945. L'idée d'un organisme français spécialement consacré à l'énergie atomique (énergie nucléaire) se fait jour. Après les bombes atomiques sur Hiroshima et Nagasaki en août 1945, de Gaulle fait adopter, en octobre 1945, l'ordonnance créant le Commissariat à l'énergie atomique; le texte en a été préparé par F.Joliot et R. Dautry. Le 3 janvier 1946, de Gaulle nomme Frédéric Joliot haut commissaire et Raoul Dautry administrateur général.

F. Joliot lance immédiatement la prospection minière d'uranium en France. Il devait décrire ainsi, trois ans plus tard, en février 1949, le programme du nouvel organisme : "Nous partions presque de zéro au point de vue matériel et il fallait tout créer, mais l'ordonnance spéciale qui installait le Commissariat avait été prise en vue de faciliter notre tâche. Nous avons aussitôt prévu trois étapes dans le développement de l'énergie atomique et la première de celles-ci comprenait la construction d'une pile à uranium et eau lourde, de faible puissance, avec tout ce que cela comportait de constructions annexes."

Sous l'impulsion enthousiaste du savant et sous la conduite de Lew Kowarski de retour du Canada, se construit ainsi au fort de Châtillon à Fontenay-aux-Roses la pile ZOÉ (pour puissance Zéro, Oxyde d'uranium et Eau lourde). Le CEA dispose pour cela des résultats obtenus par l'équipe du Collège de France en 1939-1940, de l'uranium rapporté du Maroc, des accords passés avec la Norvège pour l'eau lourde, des connaissances apportées par les Français revenus du Canada (Kowarski, Goldschmidt, Guéron et Auger) et des éléments du rapport Smyth rendu public par les Américains dès août 1945 (Review of Modern Physics, octobre 1945). Les travaux avancent rapidement. L'uranium est traité à l'usine du Bouchet, au sud de Paris. Le 15 décembre 1948, ZOÉ, la première pile atomique française, entre en fonctionnement. Elle allait fournir des radioéléments artificiels pour les applications biologiques, permettre de former les techniciens et d'élaborer les matériaux nécessaires à la construction des piles de moyenne puissance. Son installation extérieure a été conservée et on peut la visiter ${ }^{(2)}$.

\section{Bibliographie}

- Frédéric Joliot, Atomes, № 35, février 1949 et CEuvres scientifiques complètes, P.U.F., 1961, p. 822

- Michel Pinault, Frédéric Joliot-Curie, Éd. Odile Jacob, 2000

- Pierre Radvanyi, Les Curie, pionniers de l'atome, Éd. Belin, 2005.

- Spencer R. Weart, La grande aventure des atomistes français, Éd. Fayard, 1980.

\section{EXPOSITION}

"Frédéric Joliot-Curie (1900 - 1958) "

une exposition réalisée par le Musée Curie,

à Paris, Mairie du $5^{\mathrm{e}}$ arrondissement,

du 17 octobre au 6 novembre 2008.

Renseignements : 0156817505

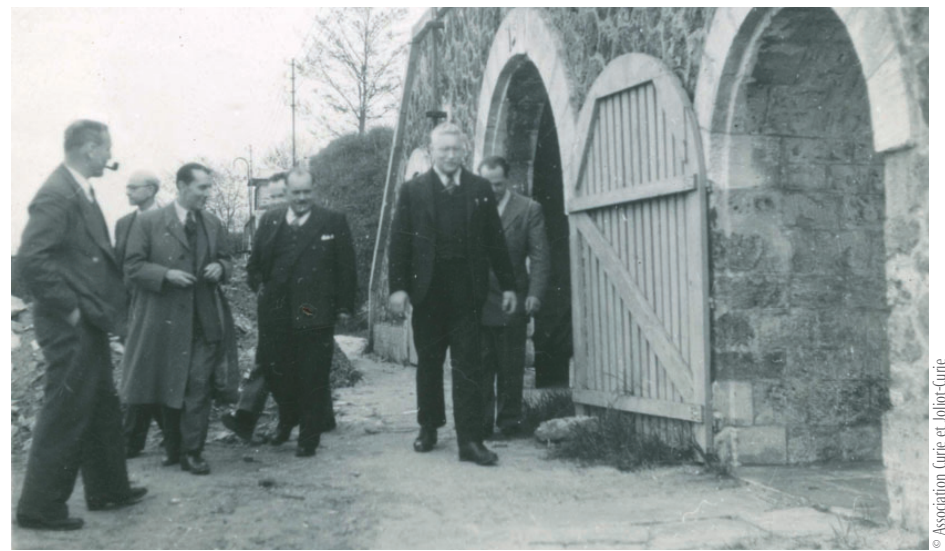

F. Joliot (en imperméable) et L. Kowarski (à droite) devant les casemates du Fort de Châtillon en 1946.

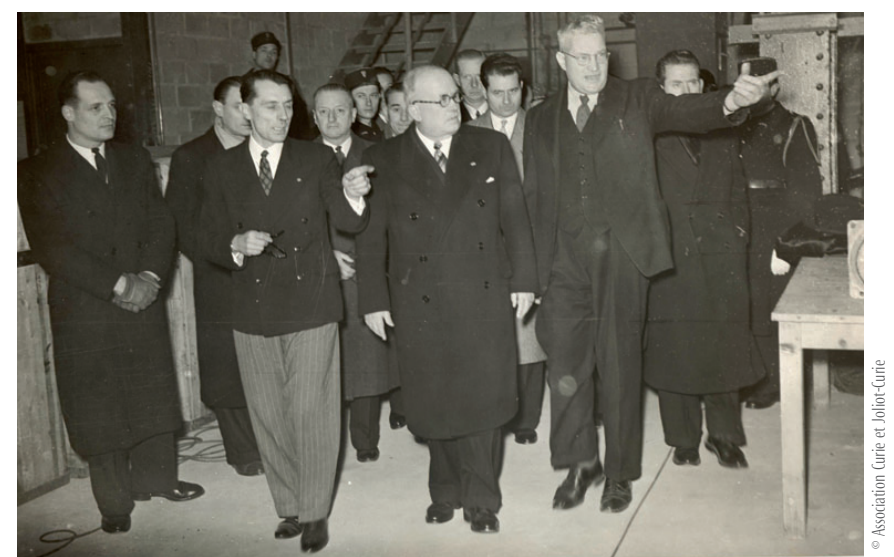

Frédéric Joliot (à gauche) et Lew Kowarski (à droite) accompagnant le président de la République, Vincent Auriol (au centre), lors de l'inauguration de Z0É en décembre 1948. 